\title{
Effect of Surface Roughness during Peristaltic Movement in a Nonuniform Channel
}

\author{
R. Shukla $\mathbb{D}^{1},{ }^{1}$ S. S. Bhatt $\mathbb{D}^{2},{ }^{2}$ A. Medhavi, ${ }^{1}$ and R. Kumar ${ }^{3}$ \\ ${ }^{1}$ Department of Mechanical Engineering, Kamla Nehru Institute of Technology, Sultanpur, India \\ ${ }^{2}$ Department of Applied Sciences and Humanities, Faculty of Engineering and Technology, Lucknow University, Lucknow, India \\ ${ }^{3}$ B. N. College of Engineering and Technology, Lucknow, India
}

Correspondence should be addressed to R. Shukla; rahul.shukla@knit.ac.in

Received 1 May 2020; Revised 12 June 2020; Accepted 25 June 2020; Published 15 July 2020

Guest Editor: Carlos Llopis-Albert

Copyright (C) 2020 R. Shukla et al. This is an open access article distributed under the Creative Commons Attribution License, which permits unrestricted use, distribution, and reproduction in any medium, provided the original work is properly cited.

In this study, the effect of the roughness parameter during the peristaltic transport of a Newtonian fluid in a nonuniform channel has been explored. The motivation of this study comes from various research studies in the area of life sciences and engineering, which reveal that the wall of living beings' arteries and all other surfaces have roughness to some extent. As peristalsis is a major mode of transporting biological fluids in various organs, the effect of surface roughness during peristaltic flow becomes very significant. The problem of peristaltic motion of a Newtonian fluid through a rough nonuniform channel having sinusoidalshaped roughness has been investigated in the current work. To analyze the flow, analytic formulation of pressure rise, friction force, velocity, and pressure gradient has been carried out under the low Reynolds number and long-wavelength approximation. Results obtained for zero surface roughness from the current model are in complete agreement with previous studies available in the literature that have been carried out without considering the surface roughness of the wall. Numerical outcomes for the properties mentioned above have been plotted for analyzing the impact of roughness on the physical and flow parameters.

\section{Introduction}

For about five decades, peristaltic transport of fluids has captured the interest of researchers because of its physiological and industrial uses; as a result, extensive research has been undertaken in this area to interpret the process of peristaltic pumping in engineering and physiological applications. Peristaltic transport involves material (slurry, solid-liquid mixture, or two immiscible liquids) transfer from one point to another using a continuous wave of area contraction and expansion along the length of an expandable duct or tube. The movement of matter in many body organs such as food in the gastrointestinal tract, urine transport to the bladder by the ureter, fluid in the cervical canal, ova transportation from the ovary to the uterus, fluid flow in the ductus efferentes, lymph movement in lymphatic vessels, and the flow of blood in small blood arteries involves peristaltic transport. Many practical applications such as a heart-lung machine, which is used during bypass surgery, and hemodialysis system involve pumping of fluid using the above mechanism. Also, this pumping mechanism is used in the pumping of corrosive chemicals, solidus liquids, slurry, and pumping of such fluids where isolation of the fluid from the moving parts of the pump is required.

To understand the mechanism of peristaltic transport, several analytical and empirical studies have been undertaken; the first in the series of studies conducted was that by Latham [1]. Shapiro et al. [2] elaborated the mechanism of peristaltic transport in detail. Gupta and Seshadri [3] examined the facts related to the peristaltic transport in nonuniform channels as well as in tubes for spermatic fluid in the vas deferens. The results of the analysis revealed that an increase in the amplitude ratio after 0.9 does not increase the flow rate; thus, peristalsis is not the only mechanism of fluid transport in the vas deferens but also some other mechanisms also exist. Major studies on peristaltic transport until 1979 have been compiled by Rath [4]. The complications of peristaltic transportation of blood flow in the 
uniform, nonuniform tube and the channel have been analyzed by Srivastava and Srivastava [5] with the presumption that blood behaves as a Casson fluid. Observation for heat transfer during transportation of the toxic liquid through pipes in a nuclear power plant has been documented by Radhakrishnamacharya and Murty [6]. Numerical analysis for stress variation during peristaltic transport was carried out by Kumar and Naidu [7]; from the study, it was concluded that the waves which possess high-amplitude and low-wave numbers produce peristaltic flows of high shear stress deviation, and such severe variation of stress can be controlled by applying an external magnitude field. Walker and Shelly [8] developed a variational technique to optimize the wave pattern for a peristaltic pump, especially for a twodimensional channel. Tripathi and Anwar Bég [9] investigated the motion of chyme across a diseased intestine; from computations, it is concluded that pressure difference and mean volumetric flow rate show an inverse relation for all parameters, but the friction force shows direct proportionality to mean volumetric flow rate. An increase in the permeability of the porous medium minimizes the pressure difference for minor values of the mean flow rate and suppresses the development of a bolus. A significant contribution to the study of heat transfer along with the effect of Hall current during peristaltic transport of the particle-fluid suspension system under the influence of the magnetohydrodynamic wall has been made by Bhatti et al. [10]. From the results obtained, the researchers concluded that reduction in fluid velocity is observed at higher values of the Hartmann number, but the fluid velocity depicts a reverse character for variation in Hall effect, particle volume fraction, wall rigidity, and wall tension parameter. An increase in the temperature profile was observed for higher values of particle volume fraction, Eckert number, and Prandtl number. Riaz et al. [11] performed an extensive computational study to investigate the behavior of nanoparticles transported by the peristaltic mechanism through a porous rectangular duct and found that increasing the value of thermophoresis parameter, volumetric flow rate, aspect ratio, local temperature, Grashof number, and porous parameter results in velocity field enlargement. The temperature profile escalates with the intensification of the Brownian motion and the thermophoresis parameter. A reverse behavior is experienced with a variation in the Brownian motion on nanoparticle concentration.

Knowledge of the effect of surface roughness during transportation of physiological fluids is very useful in understanding various problems related to blood flow in coronary arteries (Park et al. [12] and Burton and Espino [13]). Effect on flow due to surface roughness has captured the interest of researchers in chemical engineering to understand the consequence of roughness of walls formed as a result of chemical erosion during transportation of chemicals. Darcy [14] conducted experiments on 21 pipes of asphalt-covered cast iron, glass, wrought iron, and cast iron to investigate the effect of roughness of the tube during turbulent flow. The study helped to conclude that the nature of the surface, the diameter, and the slope of the pipe have a significant influence on discharge. Furthermore, it was also observed that, with an increase in the Reynolds number, the friction factor decreases, and this decrement rate gets slower for higher values of relative roughness. Holf [15] carried out an analysis for rectangular channels of unequal depths having distinct values of surface roughness and found that, during turbulent flow in pipes, mainly two types of roughness are observed which are termed surface roughness and surface corrugation, which follow different laws of similarity. Nikuradse [16] was the first to study turbulent flow at different degrees of relative roughness $(k / r)$ (where $k=$ average projection of roughness and $r=$ radius of the pipe); findings of this study helped to establish that, at a particular value of relative roughness, the velocity distribution depends slightly on the Reynolds number, but the form of this distribution strongly depends on the relative roughness. Many studies have been carried out in this area using mini channel, microchannels, and sand tubes (Kandlikar et al. [17], Wang et al. [18], Rawool et al. [19], Sun and Faghri [20], Wagner and Kandlikar [21], Lalegani et al. [22], and Ashmawy [23]). Koopaee and Zare [24] investigated the impact of interconnected and offset roughness arrangement on the fluid flow behavior along with the effect on heat transfer in microchannels with structured roughness having a sinusoidal shape.

From the above, it is concluded that though many investigations have been carried out to study the impact of surface roughness during fluid flow, no attention has been paid to find out the impact of surface roughness during peristaltic flow. In the current work, the effect of surface roughness during the peristaltic motion of a Newtonian fluid in a two-dimensional channel has been carried out under the low Reynolds number and long-wavelength approximation.

\section{Analysis}

A Newtonian fluid moving through a nonuniform channel is under investigation; furthermore, the channel wall is supposed to have sinusoidal-shaped roughness. A sinusoidal wave proliferates on channel walls with speed $c$. Assuming that the rectangular coordinate for the fixed frame is $(X, Y)$, the peristaltic transport profile is shown in Figure 1.

The geometry of the wall surface is given as

$$
H(X, t)=d(X)+b \sin \left(\frac{(2 \pi(X-c t))}{\lambda}\right)-b_{1} \cos ^{4}\left(\frac{\pi X}{\lambda_{1}}\right),
$$

with $\quad d(X)=a+K X$,

where $t$ is the time, $b$ is the amplitude of the wave, $\lambda$ is the wavelength, $a$ is the half channel width at the inlet, $K$ is the nonuniformity parameter, $c$ is the speed of wave propagation, $b_{1}$ is the roughness height, $\lambda_{1}$ is the pitch, and $X$ is the axial variable.

Continuity equation:

$$
\frac{\partial U}{\partial X}+\frac{\partial U}{\partial Y}=0
$$

Momentum equation: 


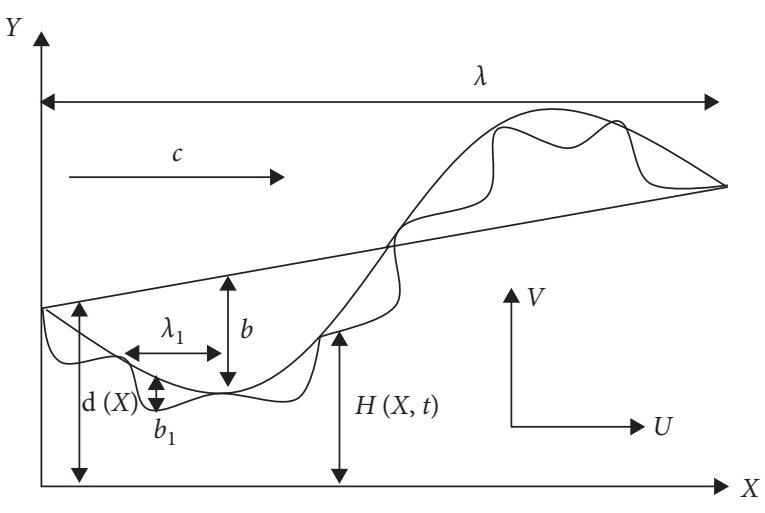

FIGURE 1: Geometry of the problem.

$$
\begin{aligned}
& \left(\frac{\partial U}{\partial t}+U \frac{\partial U}{\partial X}+V \frac{\partial U}{\partial Y}\right)=-\frac{1}{\rho} \frac{\partial p}{\partial X}+\nu\left(\frac{\partial^{2} U}{\partial X^{2}}+\frac{\partial^{2} U}{\partial Y^{2}}\right) \\
& \left(\frac{\partial V}{\partial t}+U \frac{\partial V}{\partial X}+V \frac{\partial V}{\partial Y}\right)=-\frac{1}{\rho} \frac{\partial p}{\partial Y}+\nu\left(\frac{\partial^{2} V}{\partial X^{2}}+\frac{\partial^{2} V}{\partial Y^{2}}\right)
\end{aligned}
$$

with boundary conditions

$$
\left\{\begin{array}{l}
U=0, \quad \text { at } \quad Y=H \\
\frac{\partial U}{\partial Y}=0, \quad \text { at } \quad Y=0
\end{array}\right.
$$

where $U$ and $V$ are velocity components for $X$ and $Y$ directions, respectively; in a fixed frame of reference, $p$ is the pressure, $\rho$ is the density, and $t$ is the time.

Nondimensional parameters are presented as follows:

$$
\begin{aligned}
U^{\prime} & =\frac{U}{c}, \\
V^{\prime} & =\frac{V}{c \delta}, \\
X^{\prime} & =\frac{X}{\lambda}, \\
Y^{\prime} & =\frac{Y}{a}, \\
t^{\prime} & =\frac{c t}{\lambda}, \\
p^{\prime} & =\frac{a^{2}}{c \mu \lambda}, \\
\delta & =\frac{a}{\lambda}, \\
\phi & =\frac{b}{a}, \\
\phi_{1} & =\frac{b_{1}}{a}, \\
\operatorname{Re} & =\frac{\rho a c}{\mu}, \\
\lambda_{1}^{\prime} & =\frac{\lambda_{1}}{\lambda} .
\end{aligned}
$$

Using equation (6) in equations (2) and (3) and equation (4) and dropping primes with the presumption of low Reynolds number and long-wavelength correspondence,

$$
\begin{aligned}
\frac{\partial U}{\partial X}+\frac{\partial U}{\partial Y} & =0, \\
\frac{\partial^{2} U}{\partial Y^{2}} & =\frac{\partial p}{\partial X}, \\
\frac{\partial p}{\partial Y} & =0
\end{aligned}
$$

with boundary conditions

$$
\left\{\begin{array}{l}
U=0, \quad \text { at } Y=h=\frac{H}{a}=1+\frac{K \lambda X}{a} \\
\quad+\phi \sin (2 \pi(X-t))-\phi_{1} \cos ^{4}\left(\frac{\pi X}{\lambda_{1}}\right), \\
\frac{\partial U}{\partial Y}=0, \quad \text { at } Y=0,
\end{array}\right.
$$

with $K$ which is the nonuniformity parameter of the wall, and $\phi$ is the amplitude ratio.

On solving equations (7)-(9) with given boundary condition equation (10), we get

$$
U=\left(\frac{Y^{2}-h^{2}}{2}\right) \frac{\partial p}{\partial X} .
$$

The flow flux $q(X, t)$ is computed as

$$
\begin{aligned}
q(X, t) & =\int_{0}^{h} U \mathrm{~d} Y=-\frac{h^{3}}{3} \frac{\mathrm{d} p}{\mathrm{~d} X}, \\
\text { or } \quad \frac{\mathrm{d} p}{\mathrm{~d} X} & =\frac{-3 q(X, t)}{h^{3}} .
\end{aligned}
$$

As the roughness parameter $\phi_{1} \longrightarrow 0$, the result is similar to that obtained by Gupta and Seshadri [3].

The pressure rise $\Delta p$ as well as friction force $F$ at the wall of the channel having length $L$, in their dimensionless forms, are found as

$$
\begin{aligned}
\Delta p & =\int_{0}^{(L / \lambda)}\left(\frac{\mathrm{d} p}{\mathrm{~d} X}\right) \mathrm{d} X, \\
F & =\int_{0}^{(L / \lambda)}(h)\left(-\frac{\mathrm{d} p}{\mathrm{~d} X}\right) \mathrm{d} X .
\end{aligned}
$$

\section{Results and Discussion}

Mathematica software is used to calculate the analytic results achieved from equations (14) and (15) to investigate the qualitative and quantitative effect of different parameters on the behavior of flow, pressure rise, and friction force. The instantaneous flow rate $q(X, t)$, periodic in $(X-t)$, is (Gupta and Seshadri [3]) 


$$
q(X, t)=Q+\phi \sin 2 \pi(X-t) .
$$

Following parameter values have been used: $a=0.01 \mathrm{~cm}, L=\lambda=10 \mathrm{~cm}, K=0.5(a / L)=0.0005 \quad[5,25]$, and $\phi_{1}=\left(b_{1} / a\right)$, where the value of $b_{1}$ is taken from [13].

By observing Figures 2(a) and 2(b), which depict the variation in pressure rise against time for various values of the roughness parameter for nonuniform and uniform channels, respectively, for the nonuniform channel initially, pressure rise has a significant value at the start of the peristaltic wave; however, in a narrow range of time $(t=0.2$ to 0.4$)$, a very sharp change occurs in pressure rise; this is mainly due to the result of the maximum occlusion occurring at $t=0.3$ on the entrance of the channel. The maximum pressure rise visible in the nonuniform channel increases due to a gradual increase in the roughness parameter. The results may be interpreted as with an increase in the roughness parameter, the cross-sectional area decreases; hence, channels having higher surface roughness have lesser flow area, and at the cross section of the channel, just after full occlusion, the pressure experienced is higher for high values of surface roughness due to a reduction in flow area with increasing surface roughness. For both geometries, at any instant of time, the pressure rise goes on increasing with an increase in the roughness parameter of the channel wall. Correlating Figure 2(a) with 2(b), it is concluded that the pressure rise in the uniform channel is much larger than that in a nonuniform channel.

The pressure rise against the flow rate has been plotted for different values of the roughness parameter for nonuniform as well as uniform channels in Figures 3(a) and 3(b), respectively, showing a linear relationship between pressure rise and flow rate. It is a well-known fact that pressure rise should occur in the direction of flow $(\Delta p>0)$ to use the peristaltic wave as a pump. Pressure rise increases with the time-mean flow, maximum time-mean flow occurs at zero pressure rise, and maximum pressure rise occurs at zero flow rate. However, when considering the impact of the roughness parameter of the channel wall at any value of pressure rise, there is a gain in the time-mean flow rate with an increase in the roughness parameter; also, increase in the surface roughness parameter at any flow rate results in an increase in the pressure rise. For small flow rate, a large relative change in pressure rise has been detected with increment in the surface roughness parameter. The relative change in pressure rise keeps on decreasing with an increase in flow rate and finally coincides at zero pressure rise for a uniform channel. For the nonuniform channel, pressure rise keeps on decreasing with an increase in flow rate, though the pressure rise plotted for different surface parameters comes very close but do not coincide at zero pressure rise. Similarly, for a high-pressure rise, a large relative change in flow rate has been seen with an increase in the roughness parameter; unlike the nonuniform channel, the relative change in flow rate keeps on decreasing with a decrease in the pressure rise and finally coincides at zero pressure rise for the uniform channel. On the contrary, the observed pressure rise is much smaller for the nonuniform channel than that for the uniform channel. It is also analyzed that, for zero pressure rise

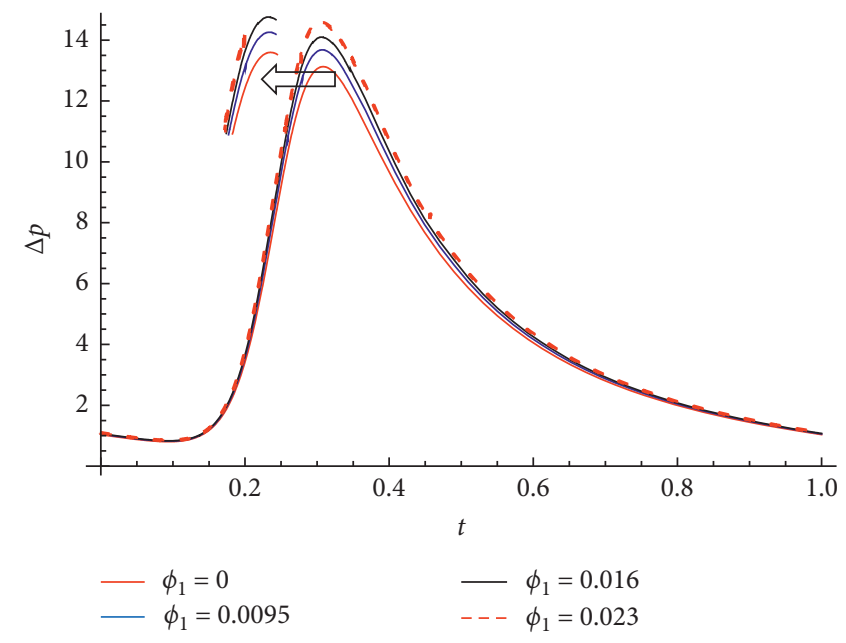

(a)

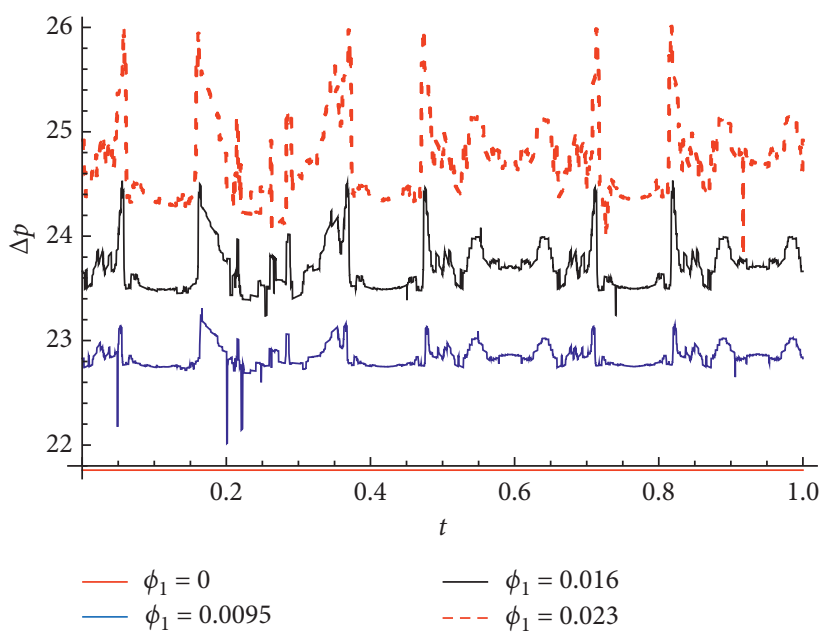

(b)

Figure 2: (a) Pressure rise versus time for various values of $\phi_{1}$ at $K=0.0005, Q=0.3, \lambda_{1}=0.00011$, and $\phi=0.8$. (b) Pressure rise versus time for various values of $\phi_{1}$ at $K=0, Q=0.3$, $\lambda_{1}=0.00011$, and $\phi=0.8$.

that is free pumping, the magnitude of the maximum rate of flow is much higher in a uniform channel than that in a nonuniform channel.

The pressure rise against the amplitude ratio has been plotted at different values of the roughness parameter in Figure 4. For a given value of surface roughness, at smaller values of the amplitude ratio, the magnitude of pressure rise increases slowly; however, at higher values of the amplitude ratio $(\phi>0.8)$, a rapid change is observed in the magnitude of pressure rise. At lower values of the amplitude ratio, a small change is observed in pressure rise with a gradual increase in the value of the roughness parameter, but rapid change in pressure rise is visible with a gradual increase in the roughness parameter at a higher magnitude of the amplitude ratio.

In Figures 5-7, it has been found that friction force shows a reverse nature to that of pressure rise.

The axial velocity distribution in the $y$-direction has been shown for a nonuniform channel in Figure 8. It can be 


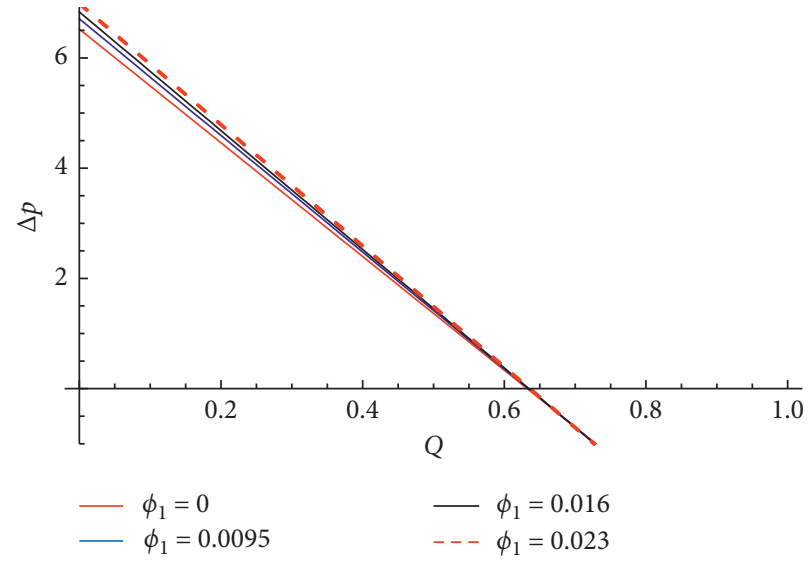

(a)

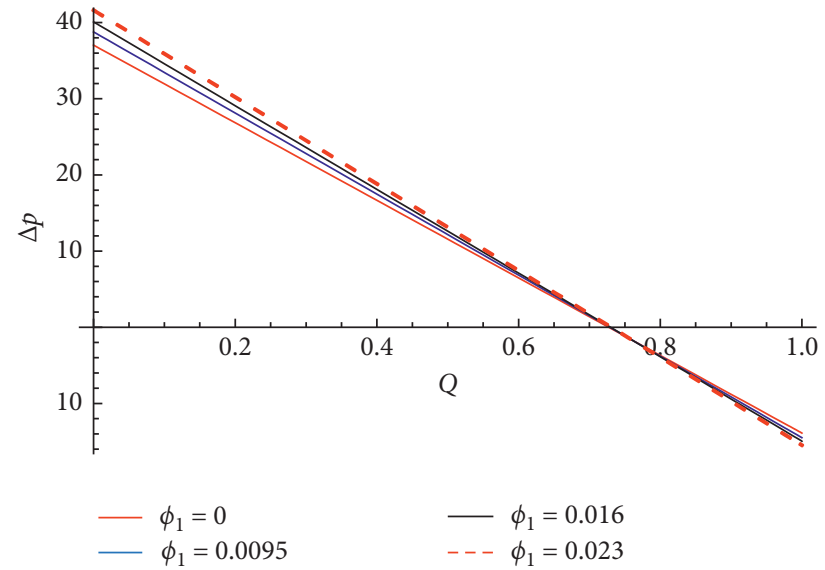

(b)

Figure 3: (a) Pressure rise versus flow rate for different values of $\phi_{1}$ at $K=0.0005, t=0.2, \lambda_{1}=0.00011$, and $\phi=0.8$. (b) Pressure rise versus flow rate for different values of $\phi_{1}$ at $K=0, t=0.2, \lambda_{1}=0.00011$, and $\phi=0.8$.

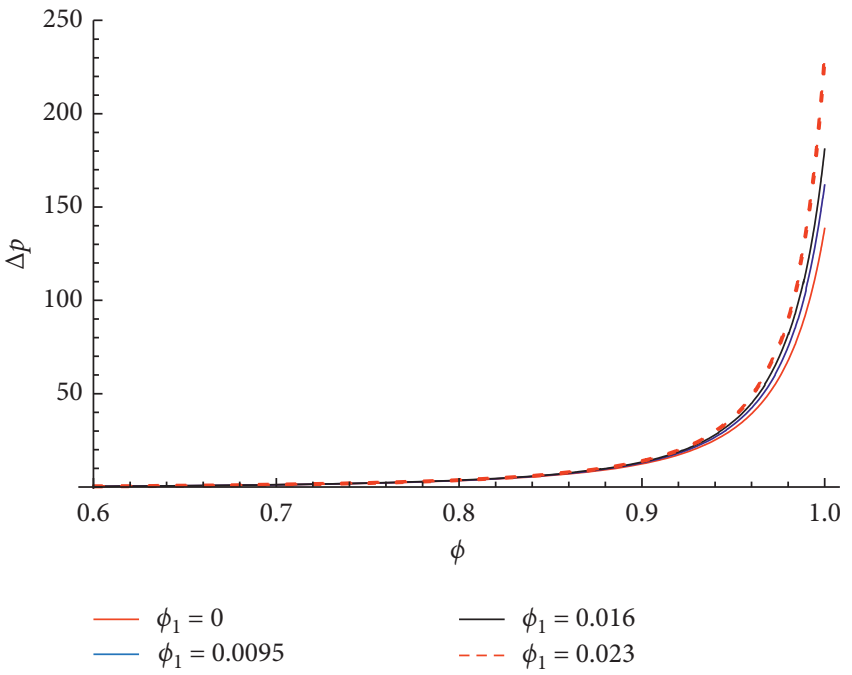

Figure 4: Pressure rise versus amplitude ratio for different values of $\phi_{1}$ at $K=0.0005, t=0.2, \lambda_{1}=0.00011$, and $Q=0.3$.

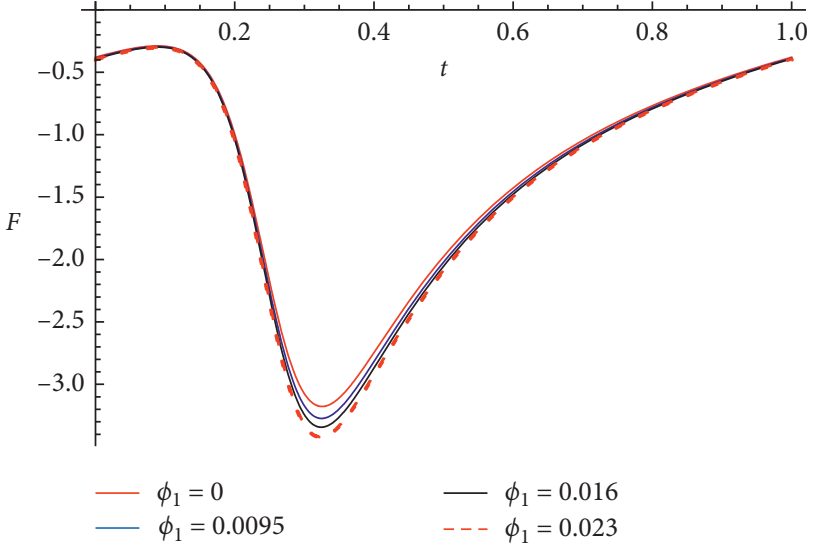

FIGURE 5: Friction force versus time for various values of $\phi_{1}$ at $K=0.0005, Q=0.3, \lambda_{1}=0.00011$, and $\phi=0.8$. inferred that the maximum velocity is obtained at the centerline for uniform as well as nonuniform channels, and it becomes zero at the wall of the channel. However, the maximum velocity of flow is observed to be higher in a uniform channel than that in a nonuniform channel. The above results can be physically interpreted as follows: pressure rise for the uniform channel is much higher than that for the nonuniform channel because in the uniform channel, full occlusion occurs throughout the channel length, whereas in the diverging channel, full occlusion occurs only at the entrance. Velocity rises with a gradual increment in the value of the roughness parameter near the center of the nonuniform channel and depicts an adverse nature in the vicinity of the wall. Similar behavior has been observed for the uniform channel. When surface roughness increases, the velocity of flow in the vicinity of the wall of the channel reduces due to an increase in the contact area of the 


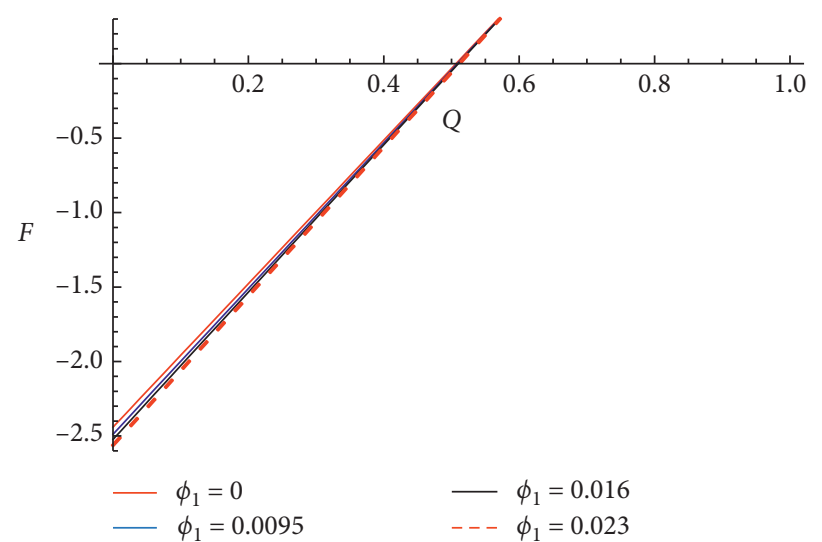

(a)

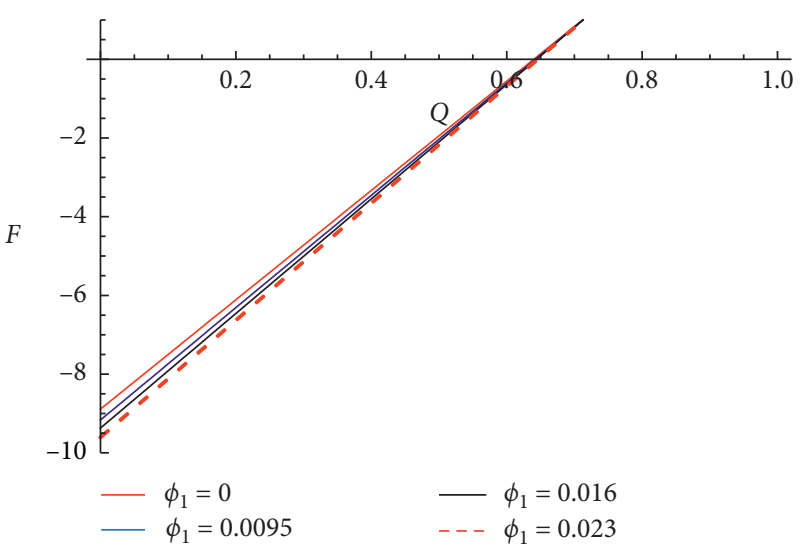

(b)

Figure 6: (a) Friction force versus flow rate for different values of $\phi_{1}$ at $K=0.0005, t=0.2, \lambda_{1}=0.00011$, and $\phi=0.8$. (b) Friction force versus flow rate for different values of $\phi_{1}$ at $K=0, t=0.2, \lambda_{1}=0.00011$, and $\phi=0.8$.

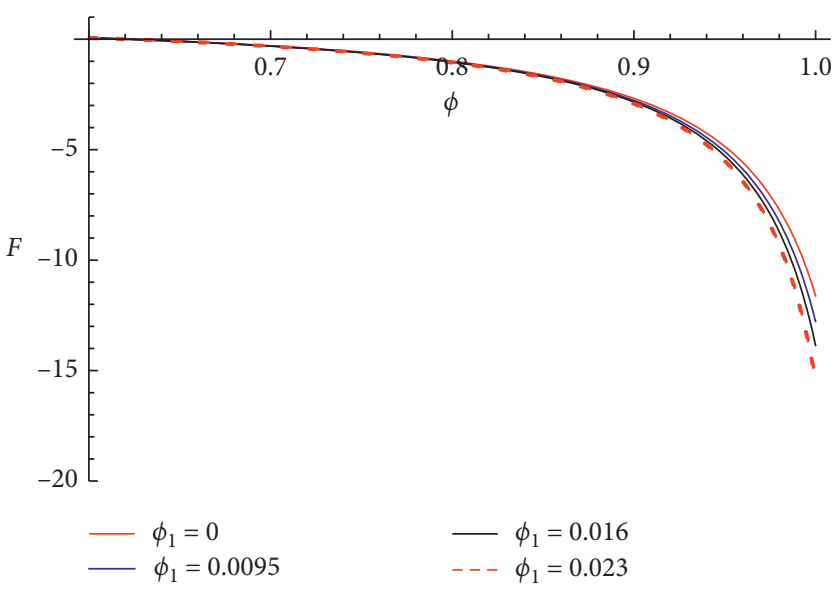

FiguRe 7: Friction force versus amplitude ratio for different values of $\phi_{1}$ at $K=0.0005, t=0.2, \lambda_{1}=0.00011$, and $Q=0.3$.

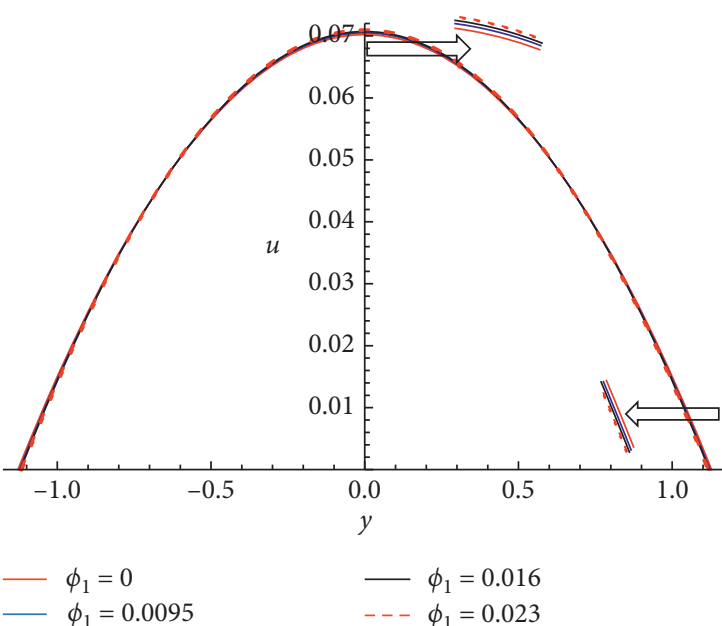

(a)

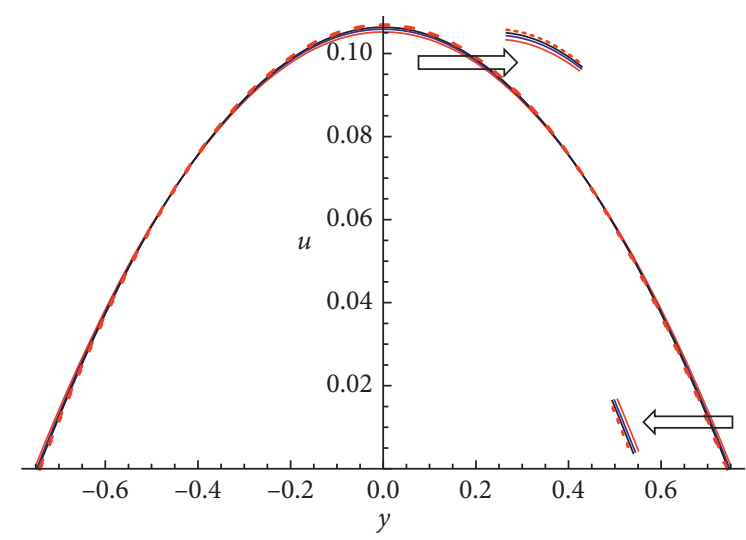

$-\phi_{1}=0$
$-\phi_{1}=0.0095$

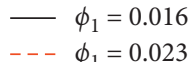

(b)

Figure 8: (a) Velocity profile for different values of $\phi_{1}$ at $K=0.0005, t=0.2, x=0.75, \lambda_{1}=0.00011$, and $Q=0.3$. (b) Velocity profile for different values of $\phi_{1}$ at $K=0, t=0.2, x=0.75, \lambda_{1}=0.00011$, and $Q=0.3$. 
fluid with the wall surface. Thus, it can be concluded that shear stress during flow increases with an increase in surface roughness. To compensate for the flow rate, there will be an increase in the magnitude of velocity close to the center of the channel.

\section{Conclusions}

The effect of surface roughness (sinusoidal structure) on peristaltic flow through a nonuniform channel for a Newtonian fluid has been studied under low Reynolds number and longwavelength approximation, and results have been compared with those obtained for a smooth channel wall. The value of maximum pressure rise increases with a gradual increase in the roughness parameter of the wall. As roughness of the surface increases, the flow area reduces, resulting in an increment in the value of maximum pressure rise. The uniform geometry with surface roughness yields much higher values of pressure rise than those for a nonuniform geometry because in the uniform channel, full occlusion occurs throughout the channel length, whereas in the diverging channel, full occlusion occurs only at the entrance. With the gradual rise in the roughness parameter, the pressure rise in the nonuniform geometry increases with a higher intensity than that in the uniform geometry. With an increase in the value of surface roughness, a decrease in the velocity of the fluid is observed near the channel wall, which is primarily due to an increase in the contact area of the fluid with the wall. Friction force shows an inverse nature to that of pressure rise. The roughness parameter shows a considerable impact on the pressure rise in the region of a small rate of flow. For the peristaltic pumping region, the roughness parameter results in an increase of flow rate for any value of pressure rise. The roughness parameter affects the magnitude of axial velocity at any cross section. Due to roughness, the magnitude of velocity close to the middle of the channel gets increased, whereas it decreases near the channel wall, showing the reverse effect of the roughness parameter on velocity. The present investigation may be helpful in future studies on the transportation of physiological fluids as the inner surface of any biological organ is not fully smooth, for example, endothelium roughness in the coronary artery.

\section{Data Availability}

The data used to support the findings of this study are available from the corresponding author upon request.

\section{Conflicts of Interest}

The authors declare that they have no conflicts of interest.

\section{References}

[1] T. W. Latham, "Fluid motion in a peristaltic pump," M. S. Thesis, Massachusetts Institute of Technology, Cambridge, Massachusetts, 1966.

[2] A. H. Shapiro, M. Y. Jaffrin, and S. L. Weinberg, "Peristaltic pumping with long wavelengths at low Reynolds number," Journal of Fluid Mechanics, vol. 37, no. 4, pp. 799-825, 1969.
[3] B. B. Gupta and V. Seshadri, "Peristaltic pumping in nonuniform tubes," Journal of Biomechanics, vol. 9, no. 2, pp. 105-109, 1976.

[4] H. J. Rath, Peristaltische Stromungen, Springer-Verlag, Berlin, Germany, 1980.

[5] L. M. Srivastava and V. P. Srivastava, "Peristaltic transport of blood: Casson model-II," Journal of Biomechanics, vol. 17, no. 11, pp. 821-829, 1984.

[6] G. Radhakrishnamacharya and V. R. Murty, "Heat transfer to peristaltic in a non uniform channel," Defence Science Journal, vol. 43, no. 3, pp. 275-280, 1993.

[7] B. V. R. Kumar and K. B. Naidu, "A numerical study of peristaltic flows," Computers \& Fluids, vol. 24, no. 2, pp. 161-176, 1995.

[8] S. W. Walker and M. J. Shelley, "Shape optimization of peristaltic pumping," Journal of Computational Physics, vol. 229, no. 4, pp. 1260-1291, 2010.

[9] D. Tripathi and O. Anwar Bég, "Peristaltic propulsion of generalized Burgers' fluids through a non-uniform porous medium: a study of chyme dynamics through the diseased intestine," Mathematical Biosciences, vol. 248, pp. 67-77, 2014.

[10] M. M. Bhatti, R. Ellahi, A. Zeeshan, M. Marin, and N. Ijaz, "Numerical study of heat transfer and Hall current impact on peristalticpropulsion of particle-fluid suspension with compliant wall properties," Modern Physics Letters B, vol. 33, no. 35, pp. 1-16, 2019.

[11] A. Riaz, A. Zeeshan, M. M. Bhatti, and R. Ellahi, "Peristaltic propulsion of Jeffrey nano-liquid and heat transfer through asymmetrical duct with moving walls in a porous medium," Physica A, vol. 545, 2020.

[12] S. W. Park, M. Intaglietta, and D. M. Tartakovsky, "Impact of endothelium roughness on blood flow," Journal of Theoretical Biology, vol. 300, pp. 152-160, 2012.

[13] H. E. Burton and D. M. Espino, "The effect of mechanical overloading on surface roughness of the coronary arteries," Applied Bionics and Biomech, vol. 2019, Article ID 2784172, 8 pages, 2019.

[14] H. Darcy, "Recherches experimentales relatives au mouvement de L'Eau dans les Tuyaux," Memoires a l'Academie d. Sciences de l'Institute imperial de France, Bd. vol. 15, p. 141, 1858.

[15] L. Holf, "Die Messung der hydraulischenRauhigkeit," ZAMMJournal of Applied Mathematics and Mechanics/Zeitschrift für Angewandte Mathematik und Mechanik, vol. 3, pp. 329-339, 1923.

[16] J. Nikuradse, "National Advisory Committee for Aeronautics; Translation of "Stromungsgesetze in rauhen Rohren," VDIForschungsheft 361," Laws of Flow in Rough Pipes. Technical Memorandum 1292, NACA, Washington, D.C., USA, 1933.

[17] S. G. Kandlikar, D. Schmitt, A. L. Carrano, and J. B. Taylor, "Characterization of surface roughness effects on pressure drop in single-phase flow in mini-channels," Physics of Fluids, vol. 17, pp. 1-11, 2005.

[18] X.-Q. Wang, C. Yap, and A. S. Mujumdar, "Effects of twodimensional roughness in flow in microchannels," Journal of Electronic Packaging, vol. 127, no. 3, pp. 357-361, 2005.

[19] A. S. Rawool, S. K. Mitra, and S. G. Kandlikar, "Numerical Simulation of flow through microchannels with designed roughness," Microfluidics and Nanofluidics, vol. 2, no. 3, pp. 215-221, 2006.

[20] H. Sun and M. Faghri, "Effect of surface roughness on nitrogen flow in a microchannel using the direct simulation Monte Carlo method," numerical heat transfer. part A: 
application," An International Journal of Computation and Methodology, vol. 43, pp. 1-8, 2010.

[21] R. N. Wagner and S. G. Kandlikar, "Effects of structured roughness on fluid flow at the microscale level," Heat Transfer Engineering, vol. 33, no. 6, pp. 483-493, 2012.

[22] F. Lalegani, M. R. Saffarian, A. Moradi, and E. Tavousi, "Effects of different roughness elements on friction and pressure drop of laminar flow in microchannels," International Journal of Numerical Methods for Heat \& Fluid Flow, vol. 28, no. 7, pp. 1664-1683, 2018.

[23] E. A. Ashmawy, "Effects of surface roughness on a couple stress fluid flow through corrugated tube," European Journal of Mechanics-B/Fluids, vol. 76, pp. 365-374, 2019.

[24] M. K. Koopaee and M. Zare, "Effect of aligned and offset roughness patterns on the fluid flow and heat transfer within microchannels consist of sinusoidal structured roughness," International Journal of Thermal Sciences, vol. 90, pp. 9-23, 2015.

[25] K. S. Mekheimer, "Peristaltic flow of blood under effect of a magnetic field in a non-uniform channels," Applied Mathematics and Computation, vol. 153, no. 3, pp. 763-777, 2004. 